\title{
miR-1271 inhibits OSCC cell growth and metastasis by targeting ALK
}

\author{
D. J. KONG ${ }^{1, *}$, J. WANG ${ }^{2, *}$, H. Q. MA ${ }^{1, *}$, G. Y. JIANG ${ }^{1, *}$ \\ ${ }^{1}$ Department of Stomatology, Jining NO.1 People's Hospital, Jining, Shandong, 272011, P.R. China; ${ }^{2}$ Department of Stomatology, Jining stomatology \\ Hospital, Jining, Shandong, 272000, P.R. China \\ ${ }^{*}$ Correspondence: jianggy_ys@163.com \\ ${ }^{*}$ Contributed equally to this work.
}

Received October 23, 2014 / Accepted January 4, 2015

\begin{abstract}
Aberrant expression of miR-1271 plays prominent roles in progression of several cancers. However, the role of miR-1271 in oral squamous cell carcinoma (OSCC) has not been elucidated. The expression levels of miR-1271 and anaplastic lymphoma kinase (ALK) were measured by qRT-PCR in OSCC tissues and cell lines. The effects of miR-1271-mediated ALK on growth and metastasis of OSCC cells, transfected with miR-1271 with or without ALK plasmid, were assessed using MTT, colony formation, migration and invasion assays. Moreover, the target relationship between miR-1271 and ALK was assessed using luciferase reporter assay, western blotting and qRT-PCR. We found that miR-1271 was significantly decreased in OSCC tissues and cell lines. Overexpression of miR-1271 suppressed cell proliferation, colony formation, migration and invasion of OSCC cells. Furthermore, ALK was identified as a target of miR-1271, ALK overexpression remarkably attenuated the tumor suppressive effects of miR-1271 on OSCC cells. In addition, ALK was inversely correlated with miR-1271 expression in OSCC tissues. Taken together, our findings indicate that miR-1271 acts as tumor suppressor in OSCC and might be used as an therapeutic target for the development of treatment for OSCC.
\end{abstract}

Key words: miR-1271, ALK, growth, metastasis, OSCC

Oral cancer is one of the most frequent solid cancer worldwide [1], and oral squamous cell carcinoma (OSCC) constitutes approximately $90 \%$ of oral cancers. Despite the improvements in therapeutic modalities, the 5-year survival rate of OSCC patients is still around 65\% [1]. OSCC is a complex genetic disorder, and previous studies have identified that several dysregulated genes were related to the progression of OSCC, but the molecular mechanism of OSCC remains unclear. Thus, it is urgent to investigate new target for OSCC treatment.

MicroRNAs (miRNAs) are important members of noncoding RNAs (ncRNAs) that suppress target gene expression by binding to the 3'-untranslated regions (3'-UTRs) to participate in intra-cellular post-transcriptional regulation $[2,3]$. Aberrant miRNA expression has also been frequently reported in numerous tumors such as lung cancer [4], pancreatic cancer [5], oral cancer [6], and hepatocellular carcinoma [7]. Recent evidence has suggests that miRNAs play essential roles in tumor cell biological processes, including cell proliferation, differentiation, migration, and invasion [8-10]. miR-1271 is an important member of miRNAs in several cancers, while the roles of miR-1271, as a oncogene or tumor suppressor, remains controversial [11-13].

In this study, we found that miR-1271 was decreased in OSCC clinical tissues and cell lines, and miR-1271 acted as a tumor suppressor. Anaplastic lymphoma kinase (ALK) was identified as a target of miR-1271 in OSCC cells, and the tumor suppressive effects of miR-1271 on OSCC cells was attenuated by ALK overexpression. In addition, ALK was inversely correlated with miR-1271 in OSCC tissues.

\section{Materials and methods}

Tissue samples, cell lines, and transfection. A total of 20 OSCC tissue samples and matched normal tissues were surgically obtained from our department between February 2013 to July 2014 . The patients were 12 men and 8 women, with a mean age of 57 years (range, 29-78). Tissues were snapfrozen in liquid nitrogen. No patients received chemotherapy or radiotherapy prior to surgery. Both tumor and non-tumor tissues were stained with $\mathrm{H} \& \mathrm{E}$ and confirmed by two experienced pathologists. The research was approved by the Hospital 
Ethical Committee and informed consent was obtained from each patient prior to the study.

Human OSCC cell lines Tca-8113(Ninth People's Hospital, Shanghai Second Medical University) were grown in RPMI 1640 medium and SCC-4(American Type Culture Collection, ATCC) were cultered in Dulbecco's modified Eagle's medium (DMEM). HEK 293T cells were also cultured in DMEM medium. All the media were supplemented with $10 \%$ fetal bovine

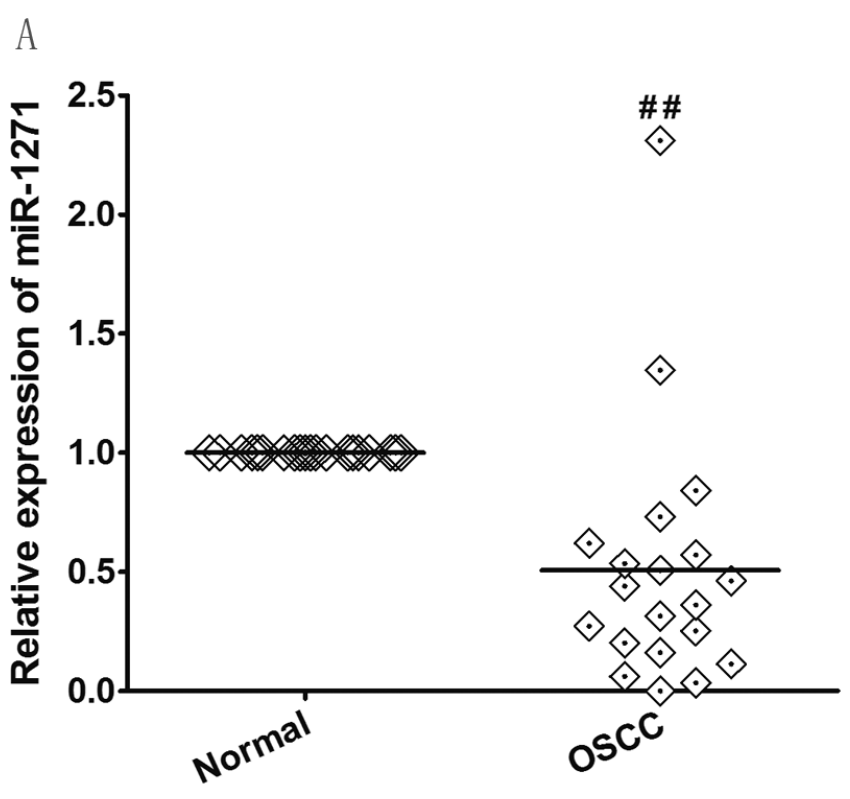

B

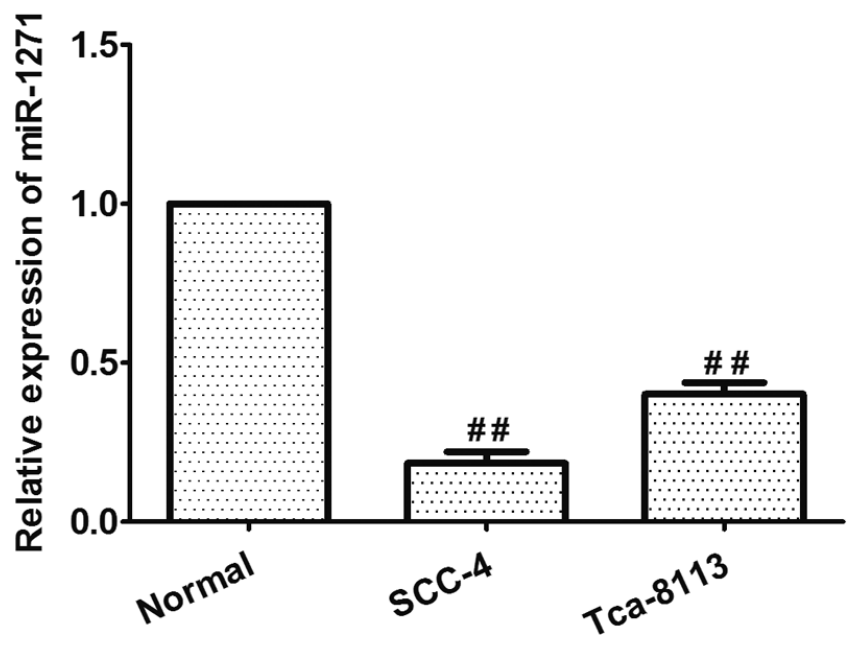

Figure 1. Down-regulation of miR-1271 in OSCC tissues and cell lines. $A$, The expression of miR-1271 in OSCC tissues and normal tissues was measured by qRT-PCR. B, The expression of miR-1271 in OSCC cell lines SCC-4, Tca-8113, and normal oral mucosa cells was measured by qRT-PCR. Experiments were performed in triplicate. ${ }^{\#} \mathbf{p}<0.01$ compared with miR-CN.
serum(FBS), $50 \mathrm{U} / \mathrm{ml}$ penicillin and $50 \mathrm{U} / \mathrm{ml}$ streptomycin at $37^{\circ} \mathrm{C}$ under $5 \% \mathrm{CO}_{2}$. Cell transfection was performed using Lipofectamine 2000 (Invitrogen) according to the manufacturer's instructions.

Vector construction. The oligonucleotides of pre-miR-1271 sence(5'-AATTCCACCCAGATCAGTGCTTGGCACCTAGCAAGCACTCAGTAAATATTTGTTGAGTGCCTGCTATGTGCCAGGCATTGTGCTGAGGGCTA-3’) and anti-sence (5'-AGCTTAGCCCTCAGCACAATGCCTGGCACATAGCAGGCACTCAACAAATATTTACTGAGTGCTTGCTAGGTGCCAAGCACTGATCTGGGTGG-3’) were synthesized and cloned into pLL3.7 plasmid. The oligonucleotides of wild-type ALK 3'-UTR (ALK-Wt) sence(5'-CGTCACGTTTTGTTTTGTGCCAACC-3'), antisence (5'-TCGAGGTTGGCACAAAACAAAACGTGACGAGCT-3') and mutation of ALK 3'-UTR (ALK-Mut) sence (5'-CGTCACGTTTTGTTTTCAGCGTTCC-3'), anti-sence(5' - TCGAGGAACGCTGAAAACAAAACGTGACGAGCT-3') were synthesized and cloned into pcDNA3.1 plasmid with luciferase reporter gene. The pcDNA3.1-ALK was generated by using the following primers: forward:5' CG TCACG T T T T G T T T TG TGCCAACC-3' and reverse:5'-TCGAGGTTGGCACAAAACAAAACGTGACGAGCT-3'.

RNA extraction and quantitative real time PCR (qRT-PCR). Total RNA was isolated by TRIzol Reagent (Invitrogen,CA, USA). miR-1271 was reversely transcribed into cDNA by special RT-primer(5'-GTCGTATCCAGTGCGTGTCGTGGAGTCGGCAATTGCACTGGATACGACTGAGTGC-3'). The qRT-PCR was performed using a SYBR Premix Ex Taq ${ }^{\mathrm{Tm}}$ II (TaKaRa) on ABI 7900. The primers for miR-1271 were forward:5'ATCCAGTGCGTGTCGTG-3', reverse: 5'-TGCTCTTGGCACCTAGCAA-3'; ALK were forward:5'-CTCAGCGAGCTGTTCAGTTG-3', reverse:5'-GGAGAAGGCATGTTTGTTGG-3'. U6 and $\beta$-actin were used to normalize miRNA and mRNA, respectively. Relative quantitation was calculated using the $2^{-\Delta \Delta \mathrm{Ct}}$ method.

Cell proliferation assay (MTT assay). Cells (4000) were seeded in 96-well plates and cultured for $24 \mathrm{~h}$ in normal conditions. Then cells were transfected with pre-miR-1271 plasmid or the control plasmid(miR-CN). The (3-4,5-dimethylthiazol2-yl)-2,5-diphenyl-tetrazolium bromide (MTT) assay was performed at 24, 48 and $72 \mathrm{~h}$, respectively. A $20 \mu \mathrm{l}$ of MTT solution was added and incubated for $4 \mathrm{~h}$ at $37^{\circ} \mathrm{C}$, then the supernatant was discarded and replaced with $150 \mu \mathrm{l}$ Dimethyl sulfoxide(DMSO). $\mathrm{OD}_{490}$ was measured using a microplate spectrophotometer.

Colony formation assay. Post-transfected cells were seeded into 12 -well plates at a density of 2000 cells/well, incubated for two weeks, and then were stained with $0.5 \%$ crystal violet for $30 \mathrm{~min}$. The pictures were obtained by using computer software.

Cell migration and invasion assays. For migration assay, the $5 \times 10^{5}$ cells were seeded into the upper chamber, and $500 \mu \mathrm{l}$ 

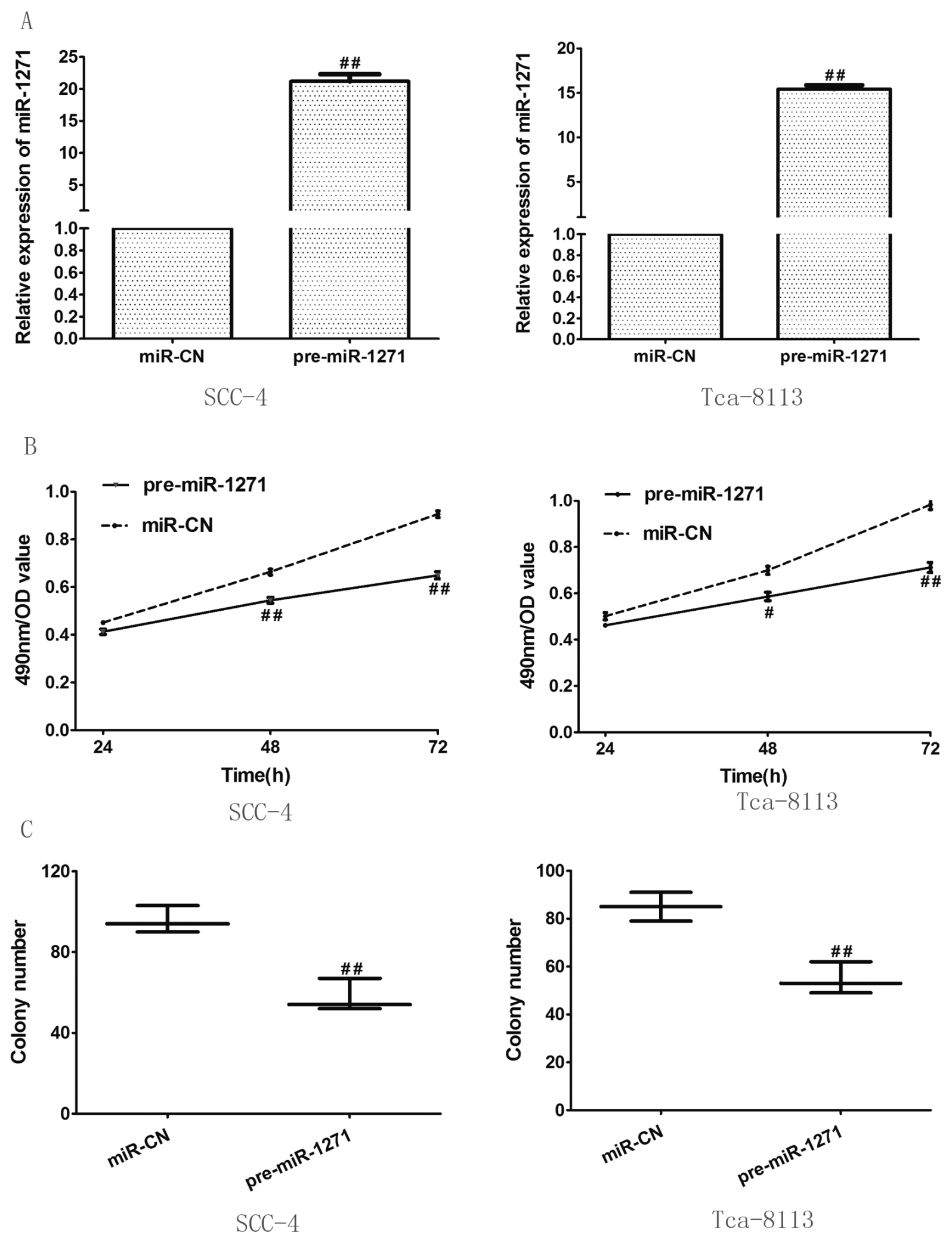

Figure 2. miR-1271 suppressed the growth of OSCC cells.

A, The expression level of miR-1271 was analyzed by qRT-PCR in SCC-4 and Tca-8113 cells transfected with pre-miR-1271 or miR-CN. B, MTT assay of SCC-4 and Tca-8113 cells transfected with pre-miR-1271 or miR-CN. C, Colony formation of SCC-4 and Tca-8113 cells transfected with pre-miR-1271 or miR-CN. Experiments were performed in triplicate. ${ }^{*} p<0.05,{ }^{* *} p<0.01$ compared with miR-CN. 
complete medium was added to the bottom chamber. Cells on the top surface of the membrane were removed using cotton swabs, and the migrated cells on the lower surfaces were fixed with $95 \%$ ethanol, and stained with $0.1 \%$ crystal violet. Matrigel-coated transwell were used for invasion assay. cells which had invaded to the lower surface of membrane were fixed, stained, and counted. Four random fields were captured for each chamber.

Luciferase reporter assay. HEK-293T cells were cotransfected with Wt or Mut of ALK and miR-1271 or miR-CN. Twenty-four hours after transfection, HEK-293T cells were
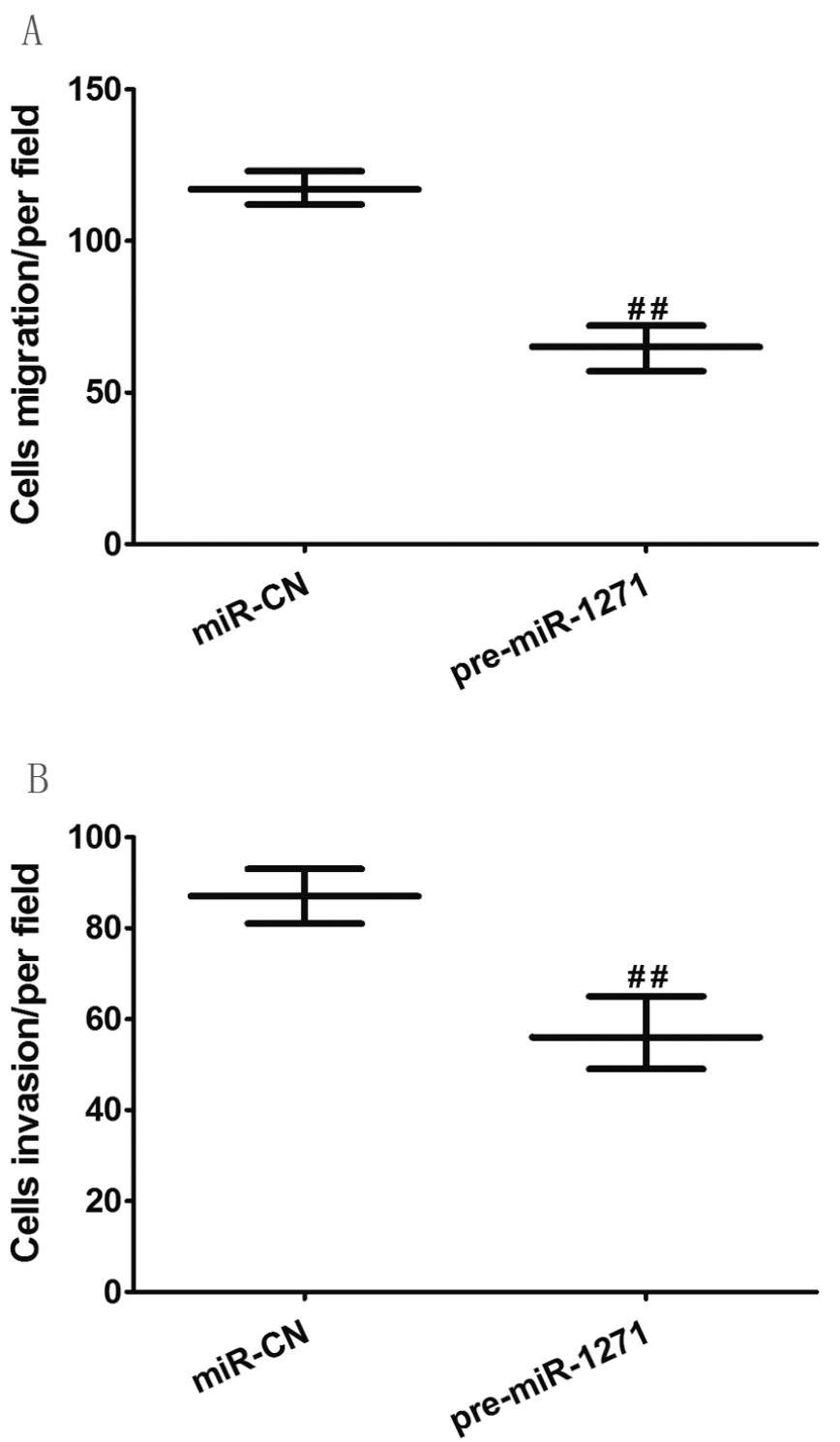

Figure 3. miR-1271 suppressed OSCC cell migration and invasion. A, Migration assay of SCC-4 and Tca-8113 cells transfected with pre-miR1271 or miR-CN. B, Invasion assay of SCC- 4 and Tca-8113 cells transfected with pre-miR-1271 or miR-CN. Experiments were performed in triplicate. ${ }^{* *} \mathbf{p}<0.01$ compared with miR-CN. harvested, and luciferase activity was assayed using a DualLuciferase Reporter Assay kit (Promega, Wisconsin, WI, USA).

Western blotting. Twenty-four hours after transfection, total protein was extracted from treated cells with RIPA buffer(Sigma-Aldrich). Then proteins were separated by $10 \%$ SDS-PAGE gel and transferred to polyvinylidene difluoride (PVDF) membrane (Millipore, MA, USA). The membrane was first incubated with specific primary antiALK(CatalogNo:60321-1-Ig, Proteintech Group Inc.), then with secondary antibody labeled with HRP and detected by chemiluminescence. The intensity was measured by ImageJ software. The protein level was normalized to $\beta$-actin.

Statistical analysis. Data were expressed as mean \pm SEM from at least three independent experiments. SPSS 13.0 analysis software was used for the statistic analysis. The differences between the two groups were analyzed using a Student's $t$ test (two-tailed). A P-value $<0.05$ was considered statistically significant.

\section{Results}

miR-1271 was downregulated in OSCC tissues and cell lines. qRT-PCR was used to detect the expression of miR-1271 in 20 OSCC tissues and two OSCC cell lines. Data showed that miR-1271 was significantly decreased in OSCC compared with normal tissues $(\mathrm{P}=0.001$, Fig. 1A). Similarly, miR-1271 was dramatically downregulated in SCC- 4 and Tca- 8113 cells compared with normal oral mucosa cells $(\mathrm{P}=0.002, \mathrm{P}=0.004$, respectively, Fig. 1B).

miR-1271 inhibited proliferation and colony formation of OSCC cells. MTT and colony formation were used to determine the effects of miR-1271 on OSCC cells. Firstly, the expression of pre-miR-1271 plasmid was identified by qRT-PCR (Fig. 2A). Then we found that pre-miR-1271 overexpression remarkably decreased the proliferation of SCC-4 and Tca-8113 cells compared with miR-CN group $(\mathrm{P}<0.05$ ,Fig. 2B). Consistent with above results, pre-miR-1271 overexpression significantly suppressed colony formation of SCC- 4 and Tca- 8113 cells $(\mathrm{P}=0.003, \mathrm{P}=0.004$, respectively, Fig. 2C).

miR-1271 suppressed migration and invasion of OSCC cells. To investigate the role of miR-1271 for the metastasis of OSCC cells, pre-miR-1271 or miR-CN was transfected into OSCC cells and migration and invasion assays were performed. As shown in Fig. 3A and B, we found that overexpression of pre-miR-1271 dramatically suppressed migration and invasion in SCC-4 and Tca-8113 cells compared with miR-CN group $(\mathrm{P}=0.001, \mathrm{P}=0.006$, respectively).

ALK was a target of miR-1271. To investigate the target of miR-1271 in OSCC, TargetScan 6.2 (http://www.targetscan. org/) was used. ALK contained the potential binding sites of miR-1271(Fig. 4A). Luciferase activity assay showed that pre-miR-1271 overexpression significantly inhibited the $\mathrm{Wt}$ 
but not the Mut luciferase activity of ALK in HEK-293T cells ( $\mathrm{P}=0.006$, Fig.4B). In addition, overexpression of pre-miR1271 significantly inhibited $\mathrm{mRNA}$ and protein levels of ALK $(\mathrm{P}<0.005$, Fig. 4C, D).

Restoration of ALK attenuated the effects of miR-1271. We further explored whether ALK overexpression could reverse the tumor-suppressive effects of miR-1271 on OSCC cells. The expression level of ALK was confirmed by qRT-PCR and western blotting(Fig. 5A, B). Furthermore, as shown in Fig. 5C, D, E, F, MTT assay, colony formation, migration and invasion showed that ALK overexpression remarkably attenuated the tumor suppressive effects of miR-1271 on OSCC cells $(\mathrm{P}<0.05)$.

ALK was inversely correlated with miR-1271 in OSCC. Expression of ALK in 20 OSCC tissues was examined by qRT-PCR. As shown in Fig. 6A, the expression of ALK was significantly increased in OSCC tissues compared with normal

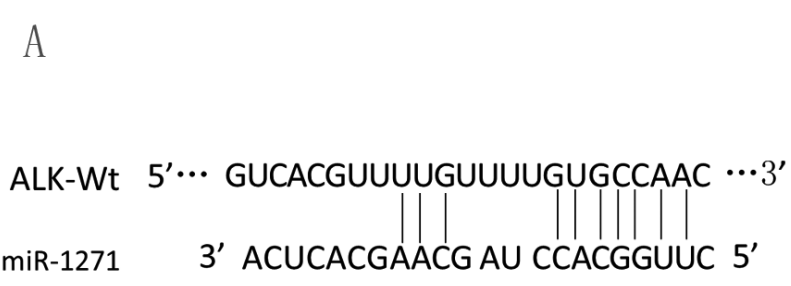

ALK-Mut 5 '... GUCACGUUUUGUUUUCAGCGUUC ... $3^{\prime}$ tissues $(\mathrm{P}<0.001)$. Moreover, ALK mRNA level was inversely correlated with miR-1271 level in OSCC tissues $(r=-0.925$, $\mathrm{P}<0.001$, Fig. 6B).

\section{Discussion}

In this study, we revealed a tumor-suppressive role of miR1271 in OSCC. In addition, we identified ALK as a direct target of miR-1271 in OSCC cells. The overexpression of ALK could attenuate suppressive effects of miR-1271 on OSCC progression. Moreover, ALK was inversely correlated with miR-1271 in OSCC tissues.

Accumulating evidence suggests the crucial role of miRNAs in OSCC progression. For example, miR-206 was found to be significantly downregulated in OSCC tissue samples and cell lines, and upregulation of miR-206 could reduce cell proliferation, invasion, and migration and promote cell apoptosis
C

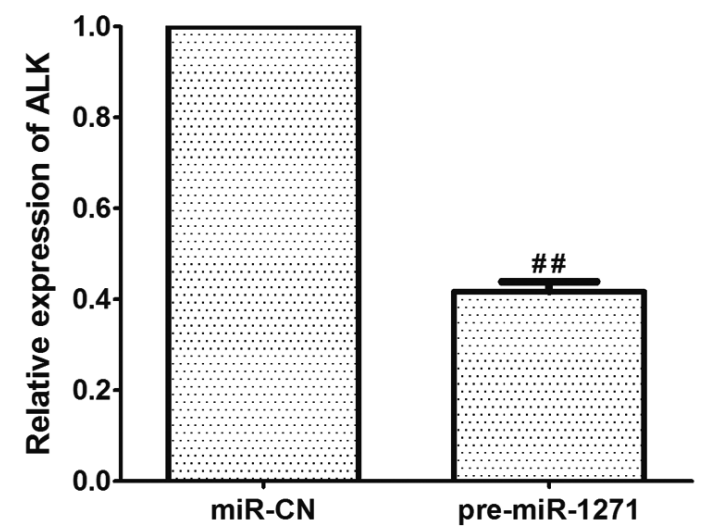

$\mathrm{B}$

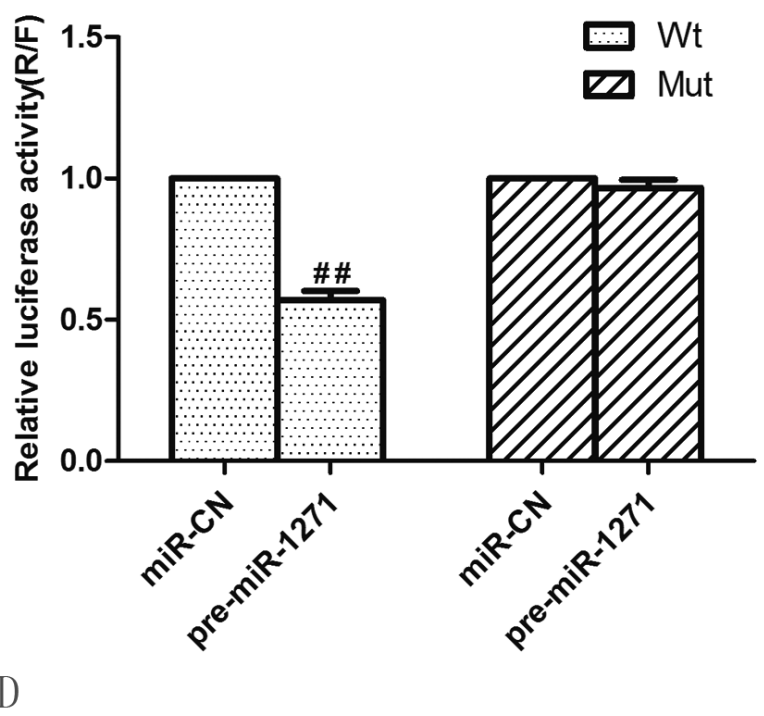

ALK

$\beta$-actin

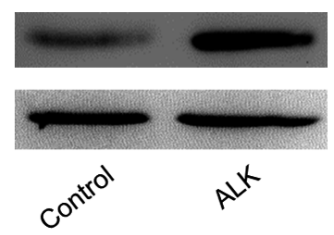

Figure 4, ALK was a direct target of miR-1271

A, The potential miR-1271 targeting site in ALK 3'-UTR and the mutated sequences. B, HEK-293T cells were co-transfected with pre-miR-1271 or miR-CN with ALK-Wt or ALK-Mut and the luciferase activity was assayed. C-D, The mRNA and protein levels of ALK were analyzed by qRT-PCR and western blotting. Experiments were performed in triplicate. ${ }^{\# \#} \mathrm{p}<0.01$ compared with miR-CN. 


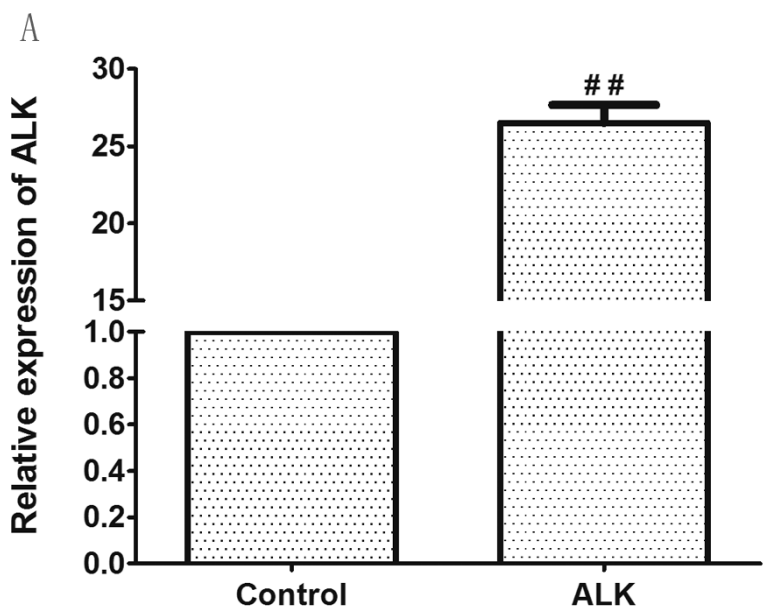

B
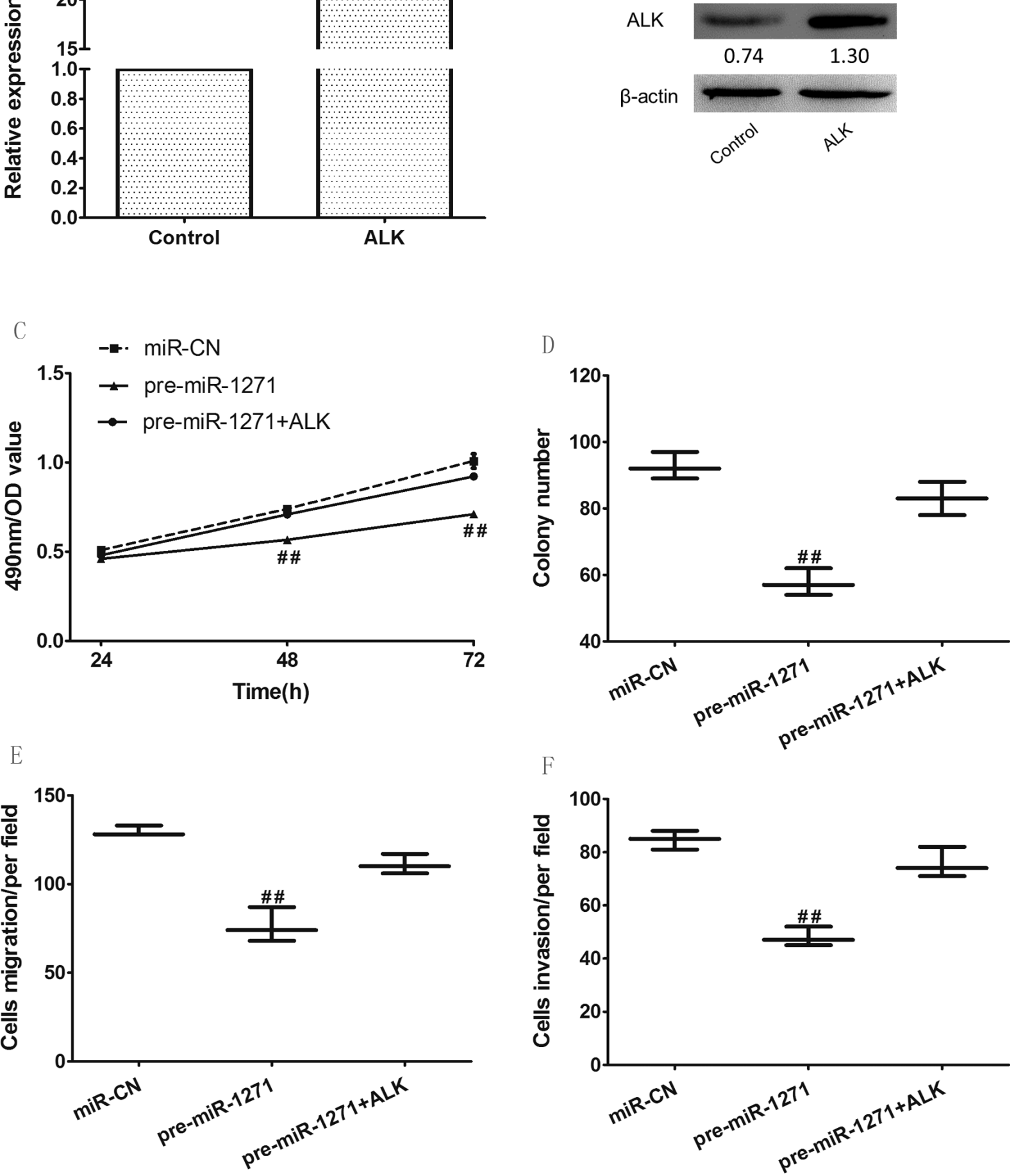

Figure 5. ALK overexpression attenuated the effects of miR-1271.

A, Expression of ALK mRNA was measured by qRT-PCR in SCC-4 cells transfected with ALK overexpression plasmid. B, ALK protein level was detected by western blotting in SCC-4 cells transfected with ALK plasmid. C, MTT assay. D, Colony formation assay. E, Migration assay. F, Invasion assay of SCC-4 cells with pre-miR-1271 with/without ALK plasmid. Experiments were performed in triplicate. ${ }^{* \#} \mathrm{p}<0.01$ compared with miR-CN. 


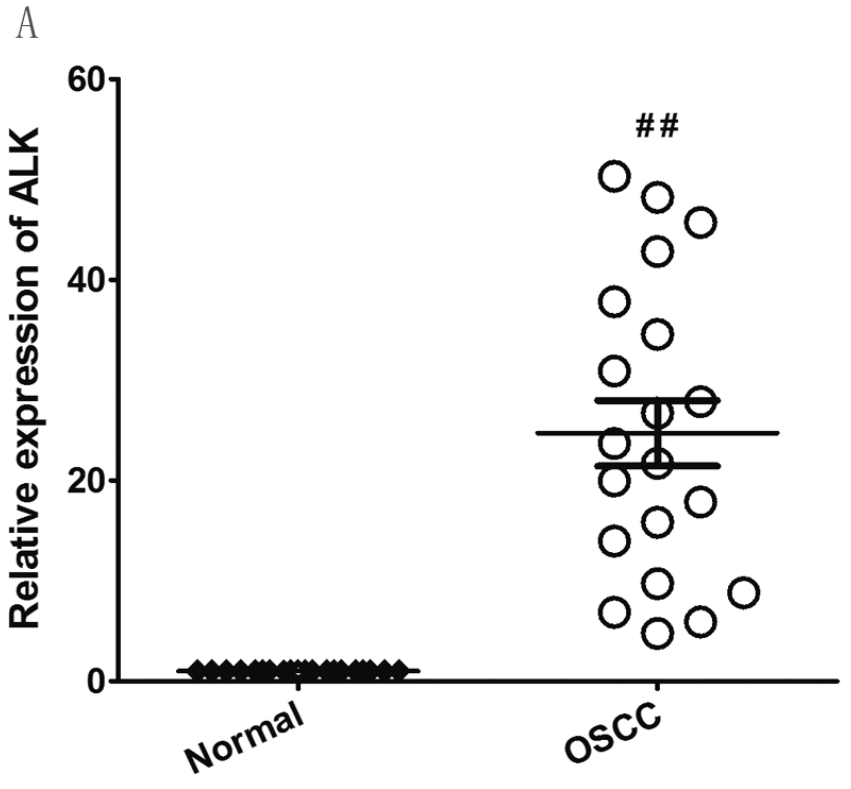

B

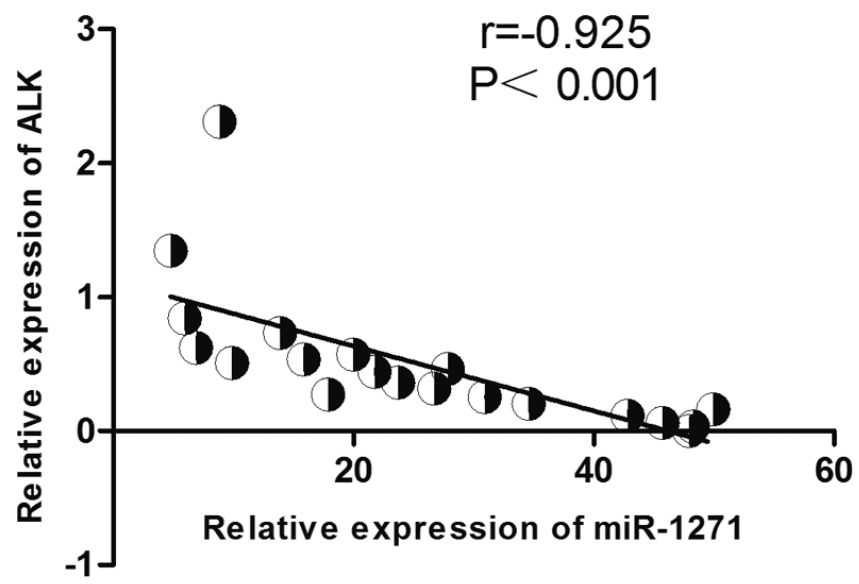

Figure 6. miR-1271 was negatively correlated with ALK in OSCC tissues. A, The expression of ALK in OSCC tissues was measured by qRT-PCR. B, ALK mRNA level was inversely correlated with miR-1271 level in OSCC tissues. Experiments were performed in triplicate. ${ }^{\# \#} p<0.01$ compared with miR-CN.

through targeting K-Ras in vitro [14]. In contrast, miR-146a was increased in OSCC tissues, and overexpression of miR146a significantly increased in vitro oncogenic phenotypes as well as during xenograft tumorigenesis and OSCC metastasis by targeting IRAK1, TRAF6 and NUMB [15]. miR-1271 has been reported to be involved in the carcinogenesis of several cancers, miR-1271 was significantly decreased in gastric cancer tissues and cell lines. Overexpression of miR-1271 inhibited proliferation of SGC7901/DDP cells, and sensitized SGC7901/DDP cells to DDP-induced apoptosis by repressed the protein levels of IGF1R, IRS1, mTOR, and BCL2[11]. Nurul-Syakima et al. [13] found that the expression of miR-1271 was up-regulated in head and neck cancers from Malaysia. Our studies revealed that the expression of miR-1271 was significantly downregulated in OSCC tissues and cells, which were in line with a previous studies $[11,12]$. Forced expression of miR-1271 suppressed proliferation, colony formation, migration and invasion, suggesting the tumor suppressive roles of miR-1271 in OSCC.

ALK is a member of the insulin receptor family of receptor tyrosine kinases and its expression is normally restricted to the nervous system [16]. Activated ALK could inhibit apoptosis and promote the proliferation through activation of down-stream PI3K/Akt and MAPK signalling pathways [17]. Accumulating evidence suggests that ALK was increased in a variety of tumors, including lung cancer [18], neuroblastoma [19], pancreatic cancer [20] and glioblastoma [21]. Martinengo et al. found that under hypoxic conditions ALK directly regulated the abundance of hypoxia-inducible factors (HIFs) in both anaplastic large cell lymphoma and non-small cell lung carcinoma. In addition. Seystahl et al. reported that TGF- $\beta$ regulates VEGF release in an ALK-5-dependent manner involving SMAD2, SMAD3, and SMAD1/5/8 signaling in glioma cells [21]. In this study, we identified that ALK was a target of miR-1271 in OSCC cells, and ALK overexpression remarkably attenuated the tumor suppressive effects of miR-1271 on OSCC cells. Furthermore, ALK was inversely correlated with miR-1271 in OSCC tissues.

In conclusion, we, for the first time, demonstrated that miR-1271 was significantly decreased in OSCC, and miR-1271 suppressed tumor growth and metastasis of OSCC cells by targeting ALK. miR-1271 might serve as a novel diagnostic marker and therapeutic target for OSCC.

Acknowledgements: This work was supported by the Project supported by the Science Foundation of the Shandong Province, China (Grant No. ZR2014HL053)

\section{References}

[1] SIEGEL R, MA J, ZOU Z, JEMAL A. Cancer statistics, 2014. CA Cancer J Clin 2014; 64: 9-29 http://dx.doi.org/10.3322/ caac. 21208

[2] BARTEL D P. MicroRNAs: target recognition and regulatory functions. Cell 2009; 136: 215-233 http://dx.doi.org/10.1016/j. cell.2009.01.002

[3] KOOIMAN G G, MARTIN F L, WILLIAMS J A, GROVER P L, PHILLIPS D H, et al. The influence of dietary and environmental factors on prostate cancer risk. Prostate Cancer Prostatic Dis 2000; 3: 256-258 http://dx.doi.org/10.1038/ sj.pcan. 4500489

[4] CHEN D Q, HUANG J Y, FENG B, PAN B Z, DE W, et al. Histone Deacetylase 1/Sp1/MicroRNA-200b Signaling Accounts for Maintenance of Cancer Stem-Like Cells in Human Lung Adenocarcinoma. PLoS One 2014; 9: e109578 
[5] KHAN S, EBELING M C, ZAMAN M S, SIKANDER M, YALLAPU M M, et al. MicroRNA-145 targets MUC13 and suppresses growth and invasion of pancreatic cancer. Oncotarget 2014; 5: 7599-7609

[6] GAO L, WANG X, WANG X, ZHANG L, QIANG C, et al. IGF-1R, a target of let-7b, mediates crosstalk between IRS-2/ Akt and MAPK pathways to promote proliferation of oral squamous cell carcinoma. Oncotarget 2014; 5: 2562-2574

[7] LI L, LIU Y, GUO Y, LIU B, ZHAO Y, et al. Regulatory miR148a-ACVR1/BMP circuit defines a cancer stem cell-like aggressive subtype of hepatocellular carcinoma. Hepatology 2014;

[8] ZHANG L, QIAN J, QIANG Y, HUANG H, WANG C, et al. Down-Regulation of miR-4500 Promoted Non-Small Cell Lung Cancer Growth. Cell Physiol Biochem 2014; 34: 1166-1174 http://dx.doi.org/10.1159/000366329

[9] JIA L F, WEI S B, MITCHELSON K, GAO Y, ZHENG Y F, et al. miR-34a Inhibits Migration and Invasion of Tongue Squamous Cell Carcinoma via Targeting MMP9 and MMP14. PLoS One 2014; 9: e108435

[10] BOYERINAS B, PARK S M, HAU A, MURMANN A E, PETER M E. The role of let-7 in cell differentiation and cancer. Endocr Relat Cancer 2010; 17: F19-36

[11] YANG M, SHAN X, ZHOU X, QIU T, ZHU W, et al. miR-1271 regulates cisplatin resistance of human gastric cancer cell lines by targeting IGF1R, IRS1, mTOR, and BCL2. Anti-cancer agents in medicinal chemistry $2014 ; 14: 884-891 \mathrm{http}$ ://dx.doi. org $/ 10.2174 / 1871520614666140528161318$

[12] MAUREL M, JALVY S, LADEIRO Y, COMBE C, VACHET $\mathrm{L}$, et al. A functional screening identifies five microRNAs controlling glypican-3: role of miR-1271 down-regulation in hepatocellular carcinoma. Hepatology 2013; 57: 195-204 http://dx.doi.org/10.1002/hep.25994

[13] NURUL-SYAKIMA A M, YOKE-KQUEEN C, SABARIAH A R, SHIRAN M S, SINGH A, et al. Differential microRNA expression and identification of putative miRNA targets and pathways in head and neck cancers. Int J Mol Med 2011; 28: 327-336

[14] LIN F, YAO L, XIAO J, LIU D, NI Z. MiR-206 functions as a tumor suppressor and directly targets K-Ras in human oral squamous cell carcinoma. OncoTargets and therapy 2014; 7: 1583-1591

[15] HUNG P S, LIU C J, CHOU C S, KAO S Y, YANG C C, et al. miR-146a enhances the oncogenicity of oral carcinoma by concomitant targeting of the IRAK1, TRAF6 and NUMB genes. PLoS One 2013; 8: e79926

[16] MORRIS S W, NAEVE C, MATHEW P, JAMES P L, KIRSTEIN M N, et al. ALK, the chromosome 2 gene locus altered by the $t(2 ; 5)$ in non-Hodgkin's lymphoma, encodes a novel neural receptor tyrosine kinase that is highly related to leukocyte tyrosine kinase (LTK). Oncogene 1997; 14: 2175-2188 http://dx.doi.org/10.1038/sj.onc.1201062

[17] POLGAR D, LEISSER C, MAIER S, STRASSER S, RUGER B, et al. Truncated ALK derived from chromosomal translocation $\mathrm{t}(2 ; 5)(\mathrm{p} 23 ; \mathrm{q} 35)$ binds to the SH3 domain of p85-PI3K. Mutat Res 2005; 570: 9-15 http://dx.doi.org/10.1016/j. mrfmmm.2004.09.011

[18] ZHOU J, YAO H, ZHAO J, ZHANG S, YOU Q, et al. Cell block from malignant pleural effusion might be a valid alternative sample for ALK detection in patients with advanced non small cell lung cancer. Histopathology 2014;

[19] MOORE N F, AZAROVA A M, BHATNAGAR N, ROSS K N, DRAKE L E, et al. Molecular rationale for the use of PI3K/ AKT/mTOR pathway inhibitors in combination with crizotinib in ALK-mutated neuroblastoma. Oncotarget 2014;

[20] YAN H H, JUNG K H, SON M K, FANG Z, KIM S J, et al. Crizotinib exhibits antitumor activity by targeting ALK signaling not c-MET in pancreatic cancer. Oncotarget 2014;

[21] SEYSTAHL K, TRITSCHLER I, SZABO E, TABATABAI G, WELLER M. Differential regulation of TGF-beta-induced, ALK-5-mediated VEGF release by SMAD2/3 versus SMAD1/5/8 signaling in glioblastoma. Neuro Oncol 2014; 